\title{
A catalogue of accurate wavelengths in the optical spectrum of the Sun $^{\star}$
}

\author{
C. Allende Prieto and R.J. García López \\ Instituto de Astrofísica de Canarias, E-38200 La Laguna, Tenerife, Spain
}

Received January 19; accepted March 18, 1998

\begin{abstract}
We present accurate measurements of the central wavelengths of 4947 atomic absorption lines in the solar optical spectrum. The wavelengths, precise to a level $\sim 50-150 \mathrm{~m} \mathrm{~s}^{-1}$, are given for both flux and disc-centre spectra, as measured in relatively recent FTS solar atlases. This catalogue modernizes existing sources based on photographic measurements and provides a benchmark to test and perform wavelength calibrations of astronomical spectra. It will also permit observers to improve the absolute wavelength calibration of solar optical spectra when lamps are not available at the telescope.
\end{abstract}

Key words: line: identification - techniques: spectroscopic - catalogs - Sun: photosphere

\section{Introduction}

Wavelength calibration is almost always needed in the process of producing useful astronomical spectra. To calibrate accurately is a non-trivial problem, in particular when working at high or very-high spectral resolution. Fourier transform spectrographs (FTS) are specially well-suited to this task, but they are not readily applied in conditions requiring high spatial or time resolution, so grating spectrometers are much more commonly used for astronomical observations. In this case, it becomes necessary to set reference positions corresponding to known wavelengths on the detector. This can be achieved by using very sharp observed telluric lines, but their location in the spectrum cannot be chosen by the astronomer. It is very usual to find spectral calibration lamps available for use with an astronomical spectrograph. The emission lines produced

Send offprint requests to: C. Allende Prieto (callende@iac.es)

* Table 1 is only available in electronic form at CDS via anonymous ftp to cdsarc.u-strasbg.fr (130.79.128.5) or via http://cdsweb.u-strasbg.fr/Abstract.html in the lamps have been previously measured at the laboratory, and this method usually provides a valid reference frame. However, it is often impractical to expose the calibration lamp simultaneously with the astronomical target and, unless the spectrograph is installed at a very stable focal station, the position of the spectrum on the detector varies depending on the telescope position. Accuracy is then limited by the instrument characteristics and observations of the calibration lamps are required between successive astronomical exposures. Nonetheless, calibrations via arc or hollow cathode spectra are normally accurate enough for most purposes.

Ingenious techniques have been used to improve the accuracy of wavelength calibrations, such as placing gas cells at the entrance of the spectrograph (e.g., Deming \& Plymate 1994), but it is rare to find such systems available and convenient for regular observations.

On occasion the available lamps are not very rich in lines in the spectral range of observation. In some circumstances, an external check of the final precision in the translation into wavelengths would be desirable. One method for tackling problems such as these is to use solar spectra as templates. Changes in the wavelengths of the lines in the integrated sunlight spectrum around the solar cycle have been proved to be very small, bellow some $15 \mathrm{~m} \mathrm{~s}^{-1}$ (Jiménez et al. 1980; Wallace et al. 1988; McMillan et al. 1993; Deming \& Plymate 1994). At $5000 \AA$, this translates into $\sim 0.3 \mathrm{m \AA}$, so the solar spectrum does offer a very stable source. In most practical cases, the accuracy will be imposed by the spectral resolution achieved. During night-time observations, the solar flux spectrum is observable after reflection from the Moon.

Measurements of solar wavelengths in the integrated solar optical spectrum were published in 1929 by Burns and collaborators (Burns 1929; Burns \& Kiess 1929; Burns \& Meggers 1929), using photographic detectors and a grating spectrograph. The relatively recent solar flux FTS atlases offer a much higher quality spectrum of the Sun seen as a star. 
As the solar spectrum is so intense, on some solar telescopes no calibration lamps are deemed necessary, and the wavelength scale is set using the solar spectrum itself. Reasonable precision can be reached using the spectrum at the centre of the disc to compare with previously measured disc centre wavelengths, thus avoiding differential shifts due to the limb effect. In this case, small scale motions have to be averaged out, integrating in time and/or space, in order to minimize errors.

The Kitt Peak Table of Photographic Solar Spectrum Wavelengths (Pierce \& Breckinridge 1973) has been extensively used by solar observers to set up the wavelength scale on their spectra. These observations, made on photographic plates, have been superseded in quality by the more recent FTS observations at the centre of the disc.

To improve on the various sets of photographically based measurements (which date back to 1930 in the case of the solar flux spectrum), provide them in a homogeneous machine-readable format, use them to test spectral calibrations of very high resolution stellar spectra (e.g., Allende Prieto et al. 1995), and improve the accuracy of our own solar observations, we have determined the position of the central wavelengths of 4947 atomic lines in the optical solar spectrum. The employed source solar atlases, prepared from FTS data, and the fashion in which we performed the measurements is described in the succeeding sections.

\section{The source atlases}

Among other solar flux FTS atlases, the Solar Flux Atlas from 296 to $1300 \mathrm{~nm}$ (Kurucz et al. 1984), which is available from the $\mathrm{NOAO}^{1} \mathrm{ftp}$ site, provided us with a highquality spectrum of the Sun seen as a star. It was obtained at the McMath ${ }^{2}$ telescope at Kitt Peak.

The FTS disc-centre spectrum included in the newer Spectral Atlas of Solar Absolute Disk-Averaged and DiskCenter Intensity from 3290 to $12510 \AA$ (Brault \& Neckel 1987; for details see Neckel 1994) was also obtained at the McMath telescope. The wavelengths of selected lines from the table of Pierce \& Breckinridge (1973), which was produced from observations with a grating spectrometer and a photographic detector at the same telescope, have been the base for placing its wavelength calibration on an absolute scale. We have measured line central wavelengths in this atlas, available to us as part of the IDL KIS ${ }^{3}$ library. A flux spectrum is also contained in this FTS atlas, which shares the source data obtained by J. Brault and collaborators with the atlas prepared by Kurucz et al. (1984).

The atlases cited achieve signal-to-noise ratios of about 2500 and a resolving power $\lambda / \Delta \lambda \sim 400000$. A quantita-

\footnotetext{
1 National Optical Astronomical Observatories, U.S.A.

2 Nowadays renamed the McMath/Pierce telescope.

3 Kiepenheuer-Institut für Sonnenphysik, Freiburg, Germany.
}

tive basis for confidence in these atlases has been established by comparison between central wavelengths of 1446 Fe I lines in the solar spectrum and at rest, performed by Allende Prieto \& García López (1998). Briefly, they found:

- The atlases have been corrected for all Doppler shifts between the centre of mass of the Sun and Earth, and the maximum shift to the red exhibited by the lines studied is the gravitational shift: $636 \mathrm{~m} \mathrm{~s}^{-1}$, corresponding to a null convective blue shift.

- There is no stronger than expected trend of the line shifts with wavelength.

- A clean correlation exists between the equivalent width and the line shift, reaching a plateau near the gravitational redshift for lines stronger than $200 \mathrm{m \AA}$.

\section{The catalogue}

This work enlarges the measurements of Allende Prieto \& García López (1998) to many other species, with the aim of optimizing its use for wavelength calibration. The line list from Thèvenin $(1989,1990)$, including 6606 lines classified by Moore et al. (1966) as singly blended or unblended, has been chosen as a guide to select the features to be measured.

A fourth-order polynomial was fitted to the $50 \mathrm{m \AA}$ wavelength interval around the line minimum to find the line centre as precisely as possible. In the electronic version of the atlases, this corresponds to 11 points for the solar flux spectrum and 25 points for the centre-of-the-disc spectrum. Errors in the wavelength determination were estimated by translating the standard deviation of the fit into the corresponding units of the wavelength axis, neglecting the extremely low photometric noise.

Table 1, available only in electronic form from the CDS, lists the central wavelengths in the flux and disccentre solar spectra, the errors of the measurements, and the element identification, excitation potential and "solar" oscillator strengths (log $g f$; from Thèvenin 1989, 1990) for 4947 lines between 3944 and $7960 \AA$ included in Thèvenin's list. More than one line corresponding to the same feature is listed when the identification is not clear from the wavelengths published by Thèvenin.

A comparison between a limited sample of wavelengths (42 lines) listed by Pierce \& Breckinridge (1973) and those we have measured in the atlas of the disc-centre shows that the absolute scales agree (difference: $79 \pm 610^{-5} \AA$ ) and that the relative differences are fully accounted for by a straightforward consideration of the expected errors in the photographic atlas $(\sim 2.5 \mathrm{m \AA})$.

Errors of the wavelengths measured in the flux spectrum can be as large as $100 \mathrm{~m} \mathrm{~s}^{-1}$, as quoted by Kurucz et al. (1984). For the wavelengths in the spectrum of the disc centre, systematic errors are given from the comparison with the photographic atlas of Pierce \& Breckinridge (1973), which has been used as reference. They estimate 
the absolute accuracy from comparison with the interferometrically determined wavelengths of Adam $(1952,1958)$ and Nichols \& Clube (1958), arriving at $0.3 \mathrm{m \AA}$. Our systematic errors are then of the order of $\sqrt{0.8^{2}+0.3^{2}} \sim$ $0.9 \mathrm{~mA}$, while FTS intrinsic errors are about $10 \mathrm{~m} \mathrm{~s}^{-1}$ (Neckel \& Labs 1990), yielding a final precision of about $50 \mathrm{~m} \mathrm{~s}^{-1}$ at $\lambda 5000 \AA$. These numbers allow us to claim that errors quoted in Table 1 (typically $\sim 50-150 \mathrm{~m} \mathrm{~s}^{-1}$ ) are conservative.

The present database updates others which were previously available based on photographic spectra, and the catalogue is presented in an homogeneous machinereadable format available via internet from the CDS. The wavelengths measured conform a reference frame which can be used to calibrate or field test calibrations of astronomical spectra by comparing with the solar spectrum, and to set up an accurate wavelength scale for solar spectra, among other applications.

Acknowledgements. We thank H. Neckel for his help in dealing with his solar atlas. We wish to extend our gratitude to F. Thévenin, who has kindly lent a digital copy of his line list, M. Collados for fruitful discussions on the wavelength calibration of solar spectra, J.E. Beckman for careful reading of the draft and H.H.R. Kroll for performing the installation and maintenance of the KIS computer libraries at the IAC. NSO/Kitt Peak FTS data used here were produced by NSF/NOAO.

This work was partially supported by the Spanish DGES under projects PB92-0434-C02-01 and PB95-1132-C02-01.
Allende Prieto C., García López R.J., Lambert D.L., Gustafsson B., 1995, in: Stellar Surface Structure, IAU Symp. 176: Poster Proceedings, Strassmeier K.G. (ed.). Vienna: Institut für Astronomie der Universität Wien, p. 107

Allende Prieto C., García López R.J., 1998, A\&AS 129, 41

Brault J., Neckel H., 1987, Spectral Atlas of Solar Absolute Disk-Averaged and Disk-Center Intensity from 3290 to $12510 \AA$, unpublished. Tape-copy from KIS IDL library

Burns K., 1929, Publ. Allegheny Obs. 6, 141

Burns K., Kiess C.C., 1929, Publ. Allegheny Obs. 6, 125

Burns K., Meggers W.F., 1929, Publ. Allegheny Obs. 6, 105

Deming D., Plymate C., 1994, ApJ 426, 382

Jímenez A., Pallé P.L., Régulo C., Roca Cortés T., Isaak G.R., McLeod C.P., van der Raay H.B., 1986, Adv. Space Res. 6,89

Kurucz R.L., Furenlid I., Brault J., Testerman L., 1984, NOAO Atlas No. 1, The Solar Flux Atlas from 296 to $1300 \mathrm{~nm}$. Sunspot, NM: National Solar Observatory

McMillan R.S., Moore T.L., Perry M.L., Smith P.H., 1993, ApJ 403, 801

Moore E., Minnaert M.G.J., Houtgast J., 1966, The Solar Spectrum $2935 \AA$ to $8770 \AA$, National Bureau of Standards Monograph 61

Neckel H., 1994, in: The Sun as a Variable Star, IAU Coll. 143, 37

Nichols S., Clube S.V.M., 1958, MNRAS 118, 496

Pierce A.K., Breckinridge J.B., 1973, Kitt Peak National Observatory, Contribution No. 559

Thèvenin F., 1989, A\&AS 77, 137

Thèvenin F., 1990, A\&AS 82, 179

Wallace L., Huang Y.R., Livingston W., 1988, ApJ 327, 399

\section{References}

Adam M.G., 1952, MNRAS 112, 546

Adam M.G., 1958, MNRAS 118, 106 